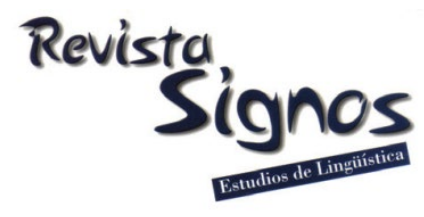

\title{
Estudio sociolingüístico de los mecanismos atenuantes utilizados en entrevistas semidirigidas de Las Palmas de Gran Canaria ${ }^{1}$
}

\author{
Sociolinguistic study of the mitigating devices used in semi- \\ structured interviews in Las Palmas de Gran Canaria
}

\author{
Marta Samper Hernández \\ Universidad de Las PaLmas de Gran CaNARIa \\ ESPAÑA \\ marta.samper@ulpgc.es
}

Recibido: 20-II-2020 / Aceptado: 11-IX-2020

DOI: $10.4067 /$ S0718-09342020000300910

\section{Resumen}

En este artículo se ofrece una relación detallada de las funciones y recursos de atenuación empleados en 18 entrevistas semidirigidas que forman parte del corpus PRESEEA-Las Palmas. Los datos se abordan tanto de manera general como a la luz de los condicionantes sociales que se han contemplado en la investigación (nivel de instrucción, edad y sexo), y también en relación con los hallados en otras comunidades de habla que ya cuentan con análisis de la atenuación dentro del proyecto PRESEEA. Los resultados permiten hablar de una cercanía entre los datos de Las Palmas de Gran Canaria y los de Santiago de Chile, frente a otras comunidades de habla como Valencia y Madrid (Vallecas), en lo que respecta a las funciones y tipos de atenuación. Por otro lado, nuestros resultados manifiestan un perfil de cambio desde abajo que refleja la difusión de la atenuación del dictum, menos frecuente entre los sujetos del nivel de instrucción alto, en la comunidad de habla estudiada. El análisis del factor sexo ofrece pocas pero claras diferencias entre hombres y mujeres.

Palabras Clave: Atenuación, PRESEEA, español de Canarias, variación diatópica, variación sociolingüística. 


\begin{abstract}
This article provides a detailed account of the mitigation functions and devices used in 18 semi-structured interviews included in the PRESEEA-Las Palmas corpus. The data are addressed both in a general way and with regard to the social variables that have been considered in the research (educational level, age and gender), and also in relation to the outcomes found in other speech communities where mitigation studies have been carried out within the PRESEEA project. With regard to the functions and types of mitigation, the results allow us to speak of a similarity between the data from Las Palmas de Gran Canaria and Santiago de Chile, compared to other speech communities such as Valencia and Madrid (Vallecas). On the other hand, our outcomes show a profile of a change from below consisting in the spread of the mitigation of the dictum, less frequent among subjects with a high level of education, in the speech community under study. Gender analysis offers few but clear differences between men and women.
\end{abstract}

Key Words: Mitigation, PRESEEA, Spanish spoken in the Canary Islands, diatopic variation, sociolinguistic variation.

\title{
INTRODUCCIÓN
}

El propósito fundamental de este artículo es ofrecer una relación detallada de los recursos lingüísticos y paralingüísticos de atenuación utilizados en 18 entrevistas semiformales pertenecientes al corpus PRESEEA de Las Palmas de Gran Canaria. Además de abordar el uso de estos mecanismos de manera general, se atenderá a su mayor o menor presencia según las principales variables sociales contempladas en el proyecto coordinado: nivel de instrucción, edad y sexo. Se trata, por tanto, de un estudio que adopta una perspectiva sociolingüística, con datos eminentemente cuantitativos sobre las tácticas empleadas. Asimismo, en consonancia con el propósito comparativo que caracteriza al proyecto en el que se inserta, se confrontarán algunos de nuestros resultados con los obtenidos en otras comunidades de habla que disponen de estudios sobre corpus paralelos.

La presente investigación aborda aspectos novedosos en relación con una serie de trabajos previos (Samper Hernández, 2013, 2017, 2018) que han puesto el foco sobre la categoría pragmática de la atenuación en el español de Las Palmas de Gran Canaria. Pese a la proliferación, en los últimos años, de investigaciones sobre la estrategia atenuadora a nivel nacional e internacional (véanse, como ejemplos, las publicaciones monográficas de Mihatsch \& Albelda (2016), Albelda \& Mihatsch, (2017)), los citados son los únicos estudios que se han realizado sobre el español isleño. Más específicamente, esta aportación completa el análisis sociolingüístico realizado en Samper Hernández (2017). En él se ofrecían los datos cuantitativos acerca del número de actos con atenuación y de la cantidad de recursos empleados para mitigar el contenido proposicional o la fuerza ilocutiva de los enunciados; en el presente artículo, como se ha dicho, se describirán las tácticas concretas que los hablantes palmenses utilizan y se prestará una especial atención a aquellas que resultan más 
frecuentes, tanto de manera general como según los diferentes condicionantes sociales.

El análisis sociolingüístico de la atenuación en un género discursivo de tipo transaccional, como son las entrevistas recogidas en los corpus PRESEEA, constituye una de las líneas de investigación del proyecto panhispánico y permite comparar los resultados de este trabajo con aquellos que han abordado este mismo tema en otras comunidades de habla. Hoy en día se dispone ya de datos procedentes de ambas orillas del Atlántico: en España se han realizado estudios sobre Madrid (Molina, 2005, 2015; Cestero, 2015, 2017), Valencia (Albelda, 2011, 2018) y Granada (Montoro, 2014; Béjar, 2015), además de los citados sobre Las Palmas de Gran Canaria; en el continente americano existen trabajos sobre Puebla (Palacios, 2017), Barranquilla (Torres \& Rodríguez, 2017), Monterrey (Flores, 2016) o Santiago de Chile (Guerrero, en prensa). Asimismo, recientemente se acaba de publicar un amplio estudio comparativo de la atenuación en cuatro comunidades de habla hispánicas: MadridVallecas, Valencia, Santiago de Chile y Las Palmas de Gran Canaria (Cestero, Albelda, Guerrero \& Samper Hernández, 2020). Sin duda, al igual que sucede en el caso de otros fenómenos lingüísticos y pragmáticos, se pueden esperar datos de gran interés a partir del análisis de la atenuación en una modalidad como la canaria, que, de acuerdo con muchos estudios previos, ocupa una posición intermedia entre las variedades peninsulares y las americanas. Recordemos, en este sentido, las palabras de Moreno (2009: 185): "El español de Canarias es el más americano de las variedades de España y la versión más española del español de América”.

El trabajo se estructura en los siguientes apartados: en primer lugar, se encuentra un marco teórico que, tras esta introducción, persigue ahondar en el objeto de estudio y la posición que ocupa en el ámbito de la investigación lingüística; a continuación, se detalla la metodología empleada en el análisis, tanto en lo que se refiere al corpus utilizado como al procedimiento seguido en el reconocimiento de los actos atenuados y de los recursos atenuadores; en tercer lugar, se exponen, analizan y discuten los resultados obtenidos; finalmente, se recogen las conclusiones más relevantes de la investigación $\mathrm{y}$, por supuesto, las referencias bibliográficas citadas a lo largo del artículo.

\section{Marco teórico}

De acuerdo con Briz y Albelda (2013: 292), consideran la atenuación como:

"una actividad argumentativa (retórica) estratégica de minimización de la fuerza ilocutiva y del papel de los participantes en la enunciación para lograr llegar con éxito a la meta prevista".

Podemos decir también, siguiendo a Briz (2007), que la atenuación es una estrategia que consiste en acercarse al otro mediante el distanciamiento con respecto al 
mensaje: el hablante se aleja del mensaje para acercarse o, al menos, para no alejarse del otro. La mitigación de la fuerza ilocutiva del acto de habla está destinada a lograr unos fines determinados, que en la inmensa mayoría de los casos -o en todos- tienen que ver con la imagen, ya sea la propia o la ajena (Albelda, 2016). Distinguimos, como Briz (2007) y Haverkate (1994), entre otros, dos niveles de incidencia de la atenuación: el dictum y el modus. De acuerdo con este planteamiento, la atenuación puede considerarse semántico-pragmática cuando afecta a lo dicho e indirectamente al decir (al modus, fuerza ilocutiva), y pragmática cuando afecta directamente al decir (Briz, 2007). Queremos subrayar, finalmente, la relación que existe entre atenuación y cortesía, dos conceptos independientes que han sido erróneamente asociados de manera biunívoca en muchas ocasiones: "Como hecho social, la atenuación es una estrategia de acercamiento a veces cortés” (Briz \& Albelda, 2013: 293).

Como se ha dicho anteriormente, en los últimos años los estudios sobre la atenuación han proliferado, de manera que esta estrategia ha sido abordada desde muy diferentes perspectivas, de las que ahora destacaremos solamente algunas. Entre otros, han sido muy frecuentes los análisis pragmáticos y sociopragmáticos de ciertos recursos o grupos de recursos mitigadores (por ejemplo, Contreras, 2018; Soler, 2018; Villalba, 2018; Flores, 2019; Repede \& León-Castro, 2019). También se han realizado estudios de tipo contrastivo entre diferentes lenguas (como los de Contreras, 2018). Del mismo modo, existen numerosos trabajos que han abordado el empleo de la estrategia atenuadora en distintos géneros discursivos, entre los que tenemos que destacar los que se centran en la conversación coloquial (Barros, 2017; Douglas, Soler \& Vuoto, 2018, entre muchos otros), pero igualmente los que parten de géneros de mayor distancia comunicativa (como los de Estellés, 2013; Villalba, 2016; Acín, 2017; Chao, 2018), en los que, en principio, se espera un mayor uso de la estrategia (Briz \& Albelda, 2013); resultan de especial interés, por supuesto, aquellos trabajos que contrastan el empleo de la atenuación en contextos de mayor y menor inmediatez comunicativa (por ejemplo, Estellés \& Cabedo, 2017-2018; Kern \& Villalba, 2017).

El tema desarrollado en el presente artículo entronca específicamente con una perspectiva de estudio que no ha sido incluida entre las anteriores, pero que ya cuenta con destacadas aportaciones. Se trata del análisis sociolingüístico, variacionista, de la atenuación, a partir de un género discursivo de menor inmediatez comunicativa que la conversación coloquial: la entrevista semidirigida. En concreto, podemos ubicar este trabajo entre los que parten de las grabaciones recogidas en el Proyecto para el estudio sociolingüistico del español de España y de América (PRESEEA), dentro del cual se contemplan distintos aspectos de la investigación lingüística: fónicos, léxicosemánticos, morfosintácticos $\mathrm{y}$, como en el caso que hoy nos ocupa, pragmáticodiscursivos. Todos los equipos trabajan de una manera coordinada, con lo que se asegura la homogeneidad entre los estudios publicados dentro del proyecto, y parten 
de unos presupuestos manifiestamente sociolingüísticos, especialmente enraizados en el variacionismo laboviano.

Aunque existe alguna publicación anterior (Molina, 2005), el tratamiento de la atenuación dentro del proyecto PRESEEA comienza 'oficialmente' en el año 2010, cuando se crea el grupo de investigación correspondiente ${ }^{2}$. Desde entonces, los análisis han proliferado y en la actualidad, como ya se ha comentado en el apartado introductorio de este artículo, se cuenta con trabajos sociolingüísticos sobre este aspecto en los materiales de PRESEEA en varias comunidades tanto españolas como hispanoamericanas: en España encontramos estudios sobre Madrid -especialmente el distrito de Vallecas (Cestero, 2011, 2012, 2015, 2017), pero también el Barrio de Salamanca (Molina, 2005, 2015)-, Valencia (Albelda, 2011, 2012, 2013, 2018), Las Palmas de Gran Canaria (Samper Hernández, 2013, 2017, 2018) y Granada (Montoro, 2014; Béjar, 2015). En América la investigación más amplia desde el punto de vista sociolingüístico es la de Santiago de Chile (Guerrero, en prensa). Indudable interés revisten los trabajos comparativos, como los que han realizado Cestero y Albelda (2012), Albelda y Briz (2010), o el de Cestero et al. (2020), que tiene en cuenta cuatro de las comunidades citadas: Madrid-Vallecas y Valencia como representantes del español centro-norteño (en comunidades monolingües y bilingües, respectivamente), Santiago de Chile como reflejo de una variedad hispanoamericana y Las Palmas de Gran Canaria como puente entre ambos lados del Atlántico.

Como afirman Briz y Albelda (2013: 292), la atenuación es mayoritariamente "utilizada en contextos situacionales de menos inmediatez o que requieren o se desea presenten menos inmediatez comunicativa", de manera que los materiales de PRESEEA, compuestos por entrevistas semidirigidas con grabadora a la vista, se convierten en instrumentos de gran utilidad para observar el empleo de esta estrategia en un género discursivo conversacional pero no coloquial. El deseo de confrontar el uso de los mecanismos atenuadores en este tipo de intercambios de carácter fundamentalmente transaccional con el que se produce en la conversación espontánea (eminentemente interpersonal) ha dado ya algunos frutos, como son las aportaciones de Estellés y Cabedo (2017-2018) o Flores (2017). Es nuestra intención, en un futuro próximo, llevar a cabo esta misma comparación también en Las Palmas de Gran Canaria, de manera que se obtenga una visión global del empleo de la atenuación en la conversación (más o menos espontánea) de los hablantes palmenses.

\section{Marco metodológico}

Para estudiar los recursos de atenuación utilizados por los hablantes palmenses se ha partido de una muestra conformada por 18 entrevistas semiformales, del total de las 72 que fueron recogidas por el equipo de Las Palmas en el marco del proyecto PRESEEA. Los informantes aparecen distribuidos de manera uniforme entre las diferentes variables independientes que se han tenido en cuenta para el análisis: hay 6 
hablantes de cada generación (la primera entre los 20 y los 34 años, la segunda entre los 35 y los 54, y la tercera a partir de los 55); 9 de los sujetos son mujeres y otros 9 son hombres; con respecto a la variación diastrática, hay que recordar que el PRESEEA atiende únicamente al nivel de instrucción de los informantes (considerado por algunos sociolingüistas (Silva-Corvalán \& Enrique-Arias, 2017) como el factor que mejor predice las características lingüísticas de la muestra, por encima del resto de los que conforman el condicionante sociocultural en el modelo multidimensional laboviano), por lo que encontramos 6 hablantes con formación universitaria, 6 con estudios medios y 6 con estudios primarios.

El corpus analizado abarca 9 horas de grabación, suma de los 30 minutos seleccionados en cada una de las 18 entrevistas. Todos los análisis comienzan sobre el minuto 10 y terminan alrededor del 40, con el objetivo principal de recoger la parte más significativa del discurso de los informantes, pero también con el propósito de facilitar la labor comparativa con los demás equipos que se dedican al estudio de la atenuación dentro de PRESEEA y que han adoptado esta misma convención. La uniformidad metodológica $y$, por lo tanto, la fiabilidad comparativa, se pone de manifiesto, asimismo, en el hecho de que todas las entrevistas incluyen diversas secuencias discursivas. En nuestro corpus se encuentran en el intervalo de tiempo seleccionado fragmentos narrativos, descriptivos, expositivos y argumentativos. En aras también de la uniformidad metodológica con anteriores publicaciones dentro de PRESEEA, así como con otras que abordan el estudio de la atenuación desde diversos puntos de vista, se ha tomado como unidad de análisis el acto de habla, concebido como una unidad estructural monológica, jerárquicamente inferior a la intervención, de la que es su constituyente inmediato; asimismo, es la mínima unidad de acción e intención, que posee las propiedades de aislabilidad e identificabilidad en un contexto dado (Grupo Val.Es.Co., 2014).

Para la determinación de las funciones y de los recursos lingüísticos que utilizan nuestros informantes para atenuar se ha partido de la ficha propuesta por Albelda, Briz, Cestero, Kotwica y Villalba (2014), que representa el vínculo entre dos perspectivas de análisis fundamentales en la investigación lingüística contemporánea: la sociolingüística y la pragmática. Indudablemente, la interrelación entre ambas perspectivas lleva a un necesario enriquecimiento de las investigaciones sobre la atenuación. Para llegar al recuento de las funciones y mecanismos mitigadores se ha llevado a cabo, en primer lugar, el pertinente análisis cualitativo de la muestra, con la consideración de los contextos interaccionales general y concreto (Albelda et al., 2014), de manera que se han distinguido aquellos casos en los que realmente existía voluntad atenuadora de otros en los que no se percibe tal finalidad. Obsérvese el contraste en el uso del verbo 'creer' entre los fragmentos (1) y (2), ambos pertenecientes a un mismo informante de nuestro corpus ${ }^{3}$ : 
(1) y el colegio:/ pues nada/ yo creo que bonito no es (LP04H13)

(2) yo fui allí creo que con quince años/ si no recuerdo mal// y había una/ tenían una hija de quince/ otra de diecisiete/ otra de diecinueve/ otra de veintiuno/ y otra de veintitrés (1") ¿sabes? (LP04H13)

En (1) el hablante se refiere a su colegio de la infancia, con el que sigue vinculado en cierta manera, y que, además, es también el de la encuestadora; es clara, pues, la voluntad que existe de no ser tajante, de minimizar su compromiso con lo dicho, algo que se refuerza mediante el empleo de una lítote a continuación ('bonito no es'). En (2), sin embargo, no existe motivo alguno que haga pensar en otra cosa que en la duda real del emisor al recordar la edad que tenía cuando realizó una estancia de intercambio en el extranjero, de manera que ni 'creo', ni la estructura condicional incidental ('si no recuerdo mal'), ni el apéndice confirmativo final (‘¿sabes?’) se han contabilizado como recursos atenuadores.

Una vez definidos los mecanismos utilizados en los diferentes actos de habla que presentan atenuación, se ha procedido al análisis estadístico de los resultados mediante el programa IBM SPSS 26. Para valorar la independencia entre variables categóricas se han utilizado la prueba de chi-cuadrado y el coeficiente de correlación de Spearman. Se han considerado resultados significativos si $\mathrm{p}<0,05$.

\section{Resultados}

\subsection{Funciones y recursos generales de atenuación. Comparación con otras comunidades de habla}

En el análisis de las 9 horas de grabación seleccionadas se han encontrado 538 actos de habla con atenuación y un total de 915 recursos atenuantes. La función absolutamente predominante es la autoprotección de la imagen (76\%). Le siguen ya de lejos la función preventiva, que aparece ante una potencial amenaza a la imagen, sobre todo, de una tercera persona $(19,7 \%) \mathrm{y}$, por último, la función reparadora de imagen, que aparece tan solo en el 4,3\% de los ejemplos. 


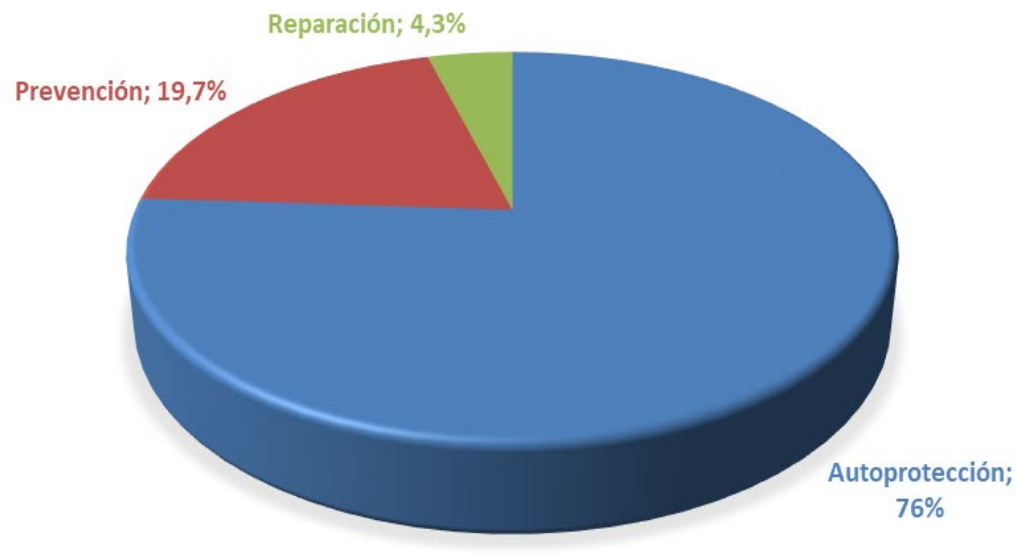

Gráfico 1. Funciones de la atenuación.

La autoprotección de la imagen la encontramos en casos como (3), en el que el entrevistado contrasta la enseñanza pública y privada de su juventud y emplea sufijos diminutivos y difusores significativos que minimizan el contenido proposicional del acto de habla y, con ello, su fuerza ilocutiva. Existe una voluntad clara de ser 'políticamente correcto' al expresar una opinión que podría resultar clasista:

(3) el el instituto era mucho más:/ justito y flojito digamos en: en lo que se refiere a la enseñanza// (LP10H23)

o también como (4), en el que un hablante joven habla sobre un hecho que ocurrió en un viaje de fin de curso y utiliza diminutivos, difusores, justificaciones y mecanismos impersonalizadores para proteger la imagen propia y de sus compañeros:

(4) salimo:s a tomar unas copillas y tal/ (ts)/ y como éramo:s chiquillos/ todavía// pue:s desde que uno se tomaba dos copas ya estaba uno: loco [de la cabeza] (LP28H12)

La función preventiva aparece mayoritariamente, como se ha apuntado, con el fin de proteger la imagen de una tercera persona. En el ejemplo (5) podemos ver cómo una madre previene la amenaza a la imagen de su hijo mediante el uso de diminutivos, cuantificadores minimizadores y difusores del significado:

(5) me dice pue:s que sí/ que lo nota un poquito más alborotado:/ como que quiere llamar un poquito más la atención// (ts) cosa que a lo mejor antes no hacía (LP55M11)

Se han hallado también 23 casos de la función reparadora. En el ejemplo (6), una empleada intenta restablecer la relación de respeto que debe existir entre ella y su jefa, tras haber afirmado que ve a esta última prácticamente de igual a igual: 
(6) I: la jefa no es como una jefa/ es como una/ una compañera más/

E: sí/

I: siempre y cuando:/ por ejemplo yo// o sea guardo las distancias/ o sea yo sé hasta dónde puedo llegar y hasta dónde no/ (LP55M11)

El predominio de la función autoprotectora coincide en prácticamente todos los trabajos que se han realizado a partir de las entrevistas semidirigidas de PRESEEA (Béjar, 2015; Cestero, 2017; Albelda, 2018; Guerrero, en prensa), lo que permite en principio corroborar la importancia del género discursivo en este aspecto. En el Gráfico 2 podemos observar cómo la frecuencia va descendiendo progresivamente desde esta función hasta la reparadora en Madrid-Vallecas, Santiago de Chile, Las Palmas y Valencia, si bien es cierto que en esta última comunidad de habla los porcentajes de la autoprotección y la prevención resultan más cercanos (existe solo un $10,7 \%$ de diferencia entre ambos). Se trata de un resultado llamativo, que podría estar relacionado con la mayor distancia comunicativa que caracteriza a las entrevistas del corpus PRESEVAL. Por otro lado, es perceptible la mayor cercanía de los perfiles de Las Palmas y Santiago de Chile: en las dos comunidades el descenso desde la función autoprotectora hasta la preventiva es mucho mayor que en las dos ciudades peninsulares. Vemos que Las Palmas estaría, como en otros casos (Samper Padilla \& Samper Hernández, 2020), en un lugar intermedio entre el español de España y el español de América, si bien más cerca de este último.

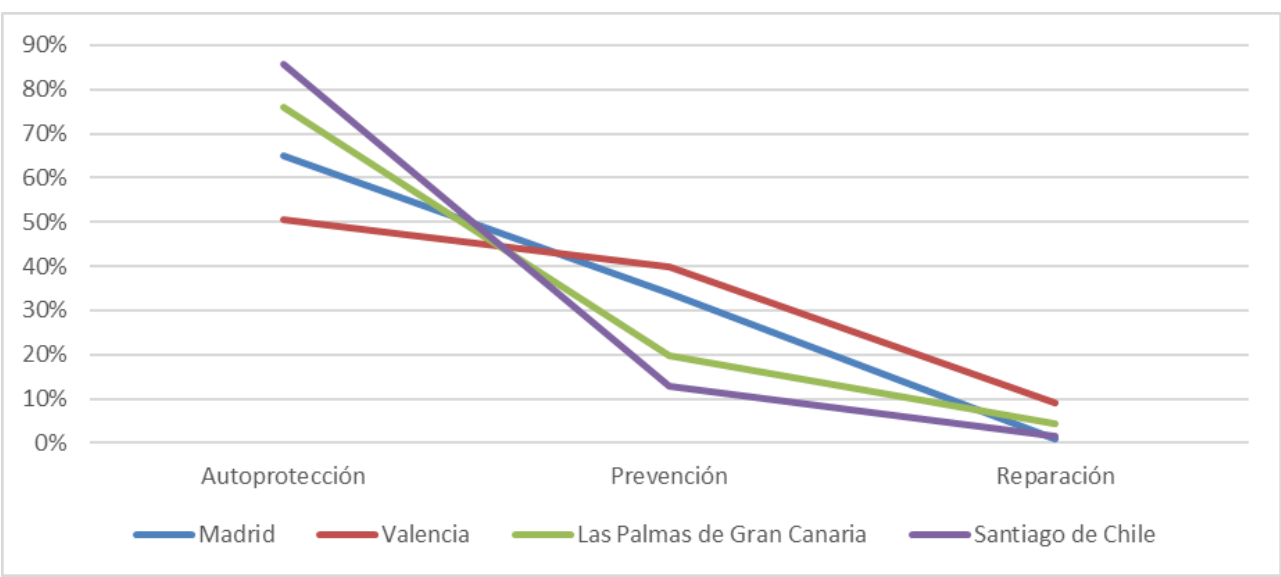

Gráfico 2. Funciones de la atenuación en cuatro comunidades de habla.

Antes de exponer los recursos concretos utilizados por los hablantes palmenses, parece interesante contemplar qué tipo de mitigación (Briz, 2007) se encuentra más frecuentemente: la que se realiza por medio de procedimientos proposicionales (sobre todo modificadores externos e internos, lítotes y eufemismos), con lo que el atenuante incide directamente sobre algún elemento del enunciado (del dictum) e indirectamente sobre la fuerza ilocutiva -como sucede en los ejemplos (3) y (5)-, la que afecta 
exclusivamente a la enunciación (al modus) -es el caso de (6) - o la que combina tácticas de ambos tipos -como se aprecia en (4)-.

En Las Palmas, en la mayoría de los casos encontrados (61,3\%) la atenuación incide directamente sobre la fuerza ilocutiva. Por otro lado, los porcentajes de actos con recursos que afectan a lo dicho y los que combinan ambos tipos de mecanismos están muy cercanos desde el punto de vista cuantitativo (20,1\% y 18,6\%, respectivamente). Son resultados que están en consonancia con los hallados en las otras comunidades analizadas dentro de PRESEEA (Cestero et al., 2020):

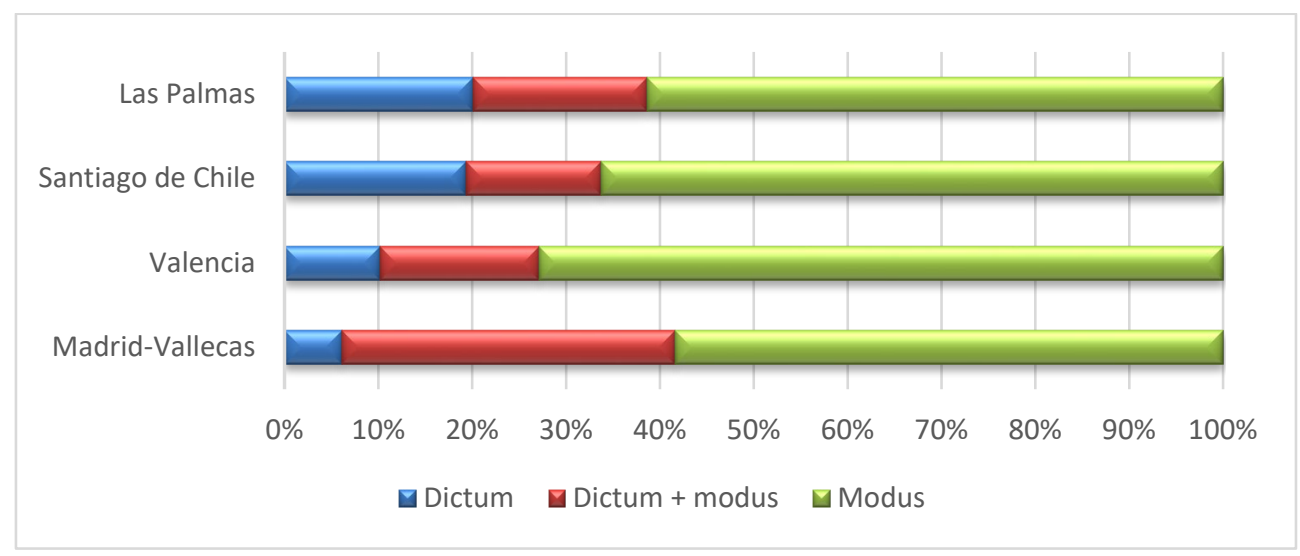

Gráfico 3. Tipos de atenuación en cuatro comunidades de habla.

Nuevamente hay que señalar que el comportamiento de Las Palmas está más cerca del de la comunidad americana: en Valencia es mayor la atenuación del modus, mientras que en Madrid-Vallecas la combinación de mecanismos de ambos tipos supera el 35\%, algo que puede deberse, ciertamente, a que en esta comunidad se dan con frecuencia dos, tres o incluso cuatro recursos por acto atenuado (Cestero, 2015; Cestero et al., 2020).

En la Tabla 1 se recogen los 21 recursos de atenuación establecidos por Albelda et al. (2014), ordenados según la frecuencia de aparición en el corpus palmense: 
Tabla 1. Recursos de atenuación ordenados según frecuencia.

\begin{tabular}{|l|l|l|}
\hline \multicolumn{1}{|c|}{ Recursos } & N & \multicolumn{1}{c|}{$\%$} \\
\hline Modificadores externos & 134 & 14,6 \\
\hline Justificaciones o excusas & 92 & 10 \\
\hline Expresiones más suaves & 69 & 7,5 \\
\hline Sufijos (diminutivos) & 65 & 7,1 \\
\hline Reformulación & 63 & 6,9 \\
\hline Elementos de control de contacto & 62 & 6,8 \\
\hline Impersonalizaciones & 61 & 6,7 \\
\hline Elementos paralingǘsticos, prosódicos y gestuales & 61 & 6,7 \\
\hline Aserciones como probabilidad/duda & 50 & 5,5 \\
\hline Concesividad & 50 & 5,5 \\
\hline Partículas evidenciales & 49 & 5,4 \\
\hline Acotación de la opinión & 48 & 5,2 \\
\hline Elipsis de la conclusión & 39 & 4,3 \\
\hline Estructuras condicionales, concesivas o temporales & 19 & 2,1 \\
\hline Fingimiento de ignorancia & 17 & 1,9 \\
\hline Impersonalizaciones mediante el estilo directo & 16 & 1,7 \\
\hline Formas de tratamiento y fórmulas apelativas & 10 & 1,1 \\
\hline Tiempos verbales & 6 & 0,7 \\
\hline Disculpas & 4 & 0,4 \\
\hline Extranjerismos & 0 & -- \\
\hline Peticiones indirectas & 0 & -- \\
\hline TOTAL & 915 & 100 \\
\hline
\end{tabular}

Como se puede comprobar, los informantes de Las Palmas de Gran Canaria emplean con más frecuencia, sobre todo, los modificadores externos, seguidos a cierta distancia, por las justificaciones o excusas, las expresiones más suaves (lítotes o eufemismos), los sufijos (diminutivos, casi en exclusividad), la reformulación, los elementos de control del contacto (‘¿no?’, ‘‘sabes?’, ‘¿me entiendes?’) y los elementos paralingüísticos, prosódicos y gestuales (fundamentalmente las risas). Los primeros cuatro recursos, que se ejemplifican a continuación, suman el 39,2\% de los casos registrados en nuestro corpus.

Entre los modificadores externos, son especialmente abundantes los cuantificadores minimizadores, que aparecen unidos en muchas ocasiones a los sufijos diminutivos ('un poquito', 'un poquillo'...), como sucede en (7), donde estos dos mecanismos se combinan también con el recurso a la risa:

(7) entonces/ yo soy un poquillo maniática// yo reconozco (risas) (LP63M21)

Además de minimizadores, también encontramos una gran cantidad de difusores del significado entre estos modificadores externos:

(8) y la mía era (1") pues una casa: (m:)// (m:) (ts) como muy alegre tenía mucha luz (LP13M23) 
Las justificaciones o excusas más habituales siguen el patrón de (9), donde un chico joven habla de un viaje turístico que hizo con su madre y otras personas mayores, e intenta salvaguardar su imagen ante lo que se puede considerar una actividad poco propia de su edad:

(9) y fuimos a montón de iglesias / que son religiosas ellas (LP49H11)

No son tan frecuentes las justificaciones del decir, aunque vemos algunos ejemplos como (10), en el que el mismo informante se refiere a su propio barrio:

(10) es un barrio:// un barrio bajo/ como se suele decir/ (LP49H11)

En lo que respecta al empleo de expresiones más suaves, predominan en nuestro corpus las lítotes sobre los eufemismos, y en el 30,4\% de los casos -muy por encima del 17,3\% de los datos generales- cumplen la función de prevenir una amenaza a la imagen de otros, como sucede claramente en (11):

(11) porque claro/ el empleado que tengo tampoco es muy:/ muy listo/ (LP34H22)

Por último, el uso de modificadores internos se limita fundamentalmente a las formas diminutivas. Además de los casos de combinación con cuantificadores minimizadores que encontramos en (5) o (7), se hallan otros ejemplos como (12), que se unen a los ya contemplados en (3) y (4):

(12) (ts) lo que pasa que el hermano ahora está en la:/ en la etapa esa de celillos también// y hace muchas cosas por llamar la atención/ cosas que quizás/ no hacía antes (LP55M11)

Algunos de los recursos más utilizados coinciden con los hallados en Madrid, Valencia o Santiago de Chile (Cestero et al., 2020), aunque en ningún caso el mecanismo más frecuente ha sido el mismo, tal como se aprecia en la Tabla 2:

Tabla 2. Recursos más frecuentes en cuatro comunidades de habla.

\begin{tabular}{|l|l|}
\hline Comunidad de habla & \multicolumn{1}{c|}{ Recurso atenuante más frecuente } \\
\hline Madrid-Vallecas & Elementos paralingüísticos \\
\hline Las Palmas & Modificadores externos \\
\hline Santiago de Chile & Justificaciones \\
\hline Valencia & Marcadores de franqueza u objetividad \\
\hline
\end{tabular}

Los modificadores externos aparecen en tercer lugar en Madrid, en el séptimo en Santiago de Chile y en el noveno en Valencia. Los elementos paralingǘsticos, que constituyen el recurso más frecuente en Madrid, no aparecen hasta la octava posición en el corpus palmense y los marcadores de franqueza y objetividad, tan frecuentes en Valencia, hasta el undécimo. Mayor cercanía encontramos, una vez más, con Santiago de Chile, ya que la recurrencia a las justificaciones y excusas como mecanismo 
atenuante ocupan en Las Palmas (también en Valencia) la segunda posición. Podemos afirmar, pues, que se trata de una táctica considerablemente generalizada en el mundo hispánico, si bien es cierto que en Madrid no figura hasta el noveno lugar de la lista (Cestero et al., 2020).

Una de las primeras conclusiones que se pueden extraer del análisis de los datos palmenses es la elevada frecuencia con que aparecen los recursos que inciden directamente sobre el contenido proposicional de los enunciados (e indirectamente, sobre la fuerza ilocutiva del acto de habla): los modificadores externos, las expresiones más suaves y los modificadores internos son tres de los cuatro mecanismos más frecuentes. Se trata de una característica diferencial con respecto a Madrid, Santiago de Chile y Valencia (Cestero et al., 2020), que presentan porcentajes mucho más bajos en el uso de los diminutivos y de los mecanismos de selección léxica (en Santiago de Chile esta última táctica no se usa en ningún caso). Debido a este carácter diferencial, y aunque ofreceremos los datos correspondientes a todos los procedimientos, nos centraremos a partir de ahora en el comentario de estos recursos.

\subsection{Recursos de atenuación y variación social}

\subsubsection{Nivel de instrucción}

Tabla 3. Mecanismos de atenuación empleados según la variable nivel de instrucción.

\begin{tabular}{|l|l|l|l|l|l|l|}
\hline \multirow{2}{*}{ Recursos } & \multicolumn{2}{|c|}{ Alto } & \multicolumn{2}{c|}{ Medio } & \multicolumn{2}{c|}{ Bajo } \\
\cline { 2 - 8 } & $\mathbf{N}$ & $\mathbf{0}$ & $\mathbf{N}$ & $\mathbf{\%}$ & \multicolumn{1}{|c|}{$\mathbf{N}$} & \multicolumn{1}{c|}{$\mathbf{0}$} \\
\hline Sufijos (diminutivos) & 20 & 5,2 & 21 & 7,1 & 24 & 10,3 \\
\hline Modificadores externos & 49 & 12,8 & 47 & 15,8 & 38 & 16,2 \\
\hline Expresiones más suaves & 27 & 7 & 24 & 8,1 & 18 & 7,7 \\
\hline Extranjerismos & 0 & -- & 0 & -- & 0 & -- \\
\hline Tiempos verbales & 3 & 0,8 & 1 & 0,3 & 2 & 0,9 \\
\hline Aserciones como probabilidad/duda & 20 & 5,2 & 21 & 7,1 & 9 & 3,8 \\
\hline Fingimiento de ignorancia & 11 & 2,9 & 3 & 1 & 3 & 1,3 \\
\hline Acotación de la opinión & 13 & 3,4 & 16 & 5,4 & 19 & 8,1 \\
\hline Peticiones indirectas & 0 & -- & 0 & -- & 0 & -- \\
\hline Disculpas & 2 & 0,5 & 2 & 0,7 & 0 & -- \\
\hline Estructuras condicionales, concesivas o temporales & 5 & 1,3 & 8 & 2,7 & 6 & 2,6 \\
\hline Justificaciones o excusas & 39 & 10,2 & 32 & 10,8 & 21 & 9 \\
\hline Elipsis de la conclusión & 16 & 4,2 & 11 & 3,7 & 12 & 5,1 \\
\hline Impersonalizaciones* & 27 & 7 & 25 & 8,4 & 9 & 3,8 \\
\hline Impersonalizaciones mediante el estilo directo & 7 & 1,8 & 4 & 1,3 & 5 & 2,1 \\
\hline Reformulación* & 35 & 9,1 & 14 & 4,7 & 14 & 6 \\
\hline Concesividad* & 15 & 3,9 & 28 & 9,4 & 7 & 3 \\
\hline Partículas evidenciales & 28 & 7,3 & 10 & 3,4 & 11 & 4,7 \\
\hline Elementos de control de contacto* & 38 & 9,9 & 14 & 4,7 & 10 & 4,3 \\
\hline Formas de tratamiento y fórmulas apelativas* & 2 & 0,5 & 1 & 0,3 & 7 & 3 \\
\hline Elementos paralingüísticos, prosódicos y gestuales & 27 & 7 & 15 & 5,1 & 19 & 8,1 \\
\hline TOTALES & 384 & 100 & 297 & 100 & 234 & 100 \\
\hline
\end{tabular}

$*$ Resultados significativos $(\mathrm{p}<0,05)$ 
En consonancia con los datos generales de atenuación, se observa que en los tres niveles de instrucción se recurre mayoritariamente a los modificadores externos. Sin embargo, las frecuencias relativas, obtenidas a partir del total de los recursos empleados en cada grupo, nos indican que estos mecanismos son más utilizados por los estratos medio $(15,8 \%)$ y bajo $(16,2 \%)$ que por los hablantes universitarios $(12,8 \%)$. Además, al contrario de lo que sucede con los otros dos grupos, los informantes del nivel bajo emplean con mayor frecuencia, tras los modificadores externos, los diminutivos, otro procedimiento que afecta al contenido proposicional del mensaje; el porcentaje $(10,3 \%)$ es, de hecho, considerablemente elevado si lo comparamos con el sociolecto medio $(7,1 \%)$ y, sobre todo, con el alto $(5,2 \%)$. El tercer mecanismo atenuador del dictum, el empleo de formas más suaves como las lítotes o los eufemismos, aparece en proporciones similares en los tres grupos.

Como se puede observar, la suma de las frecuencias relativas de los tres procedimientos mitigadores del contenido proposicional de los enunciados va siendo mayor a medida que desciende el nivel de instrucción de los sujetos entrevistados: $25 \%$ en el nivel alto, $31 \%$ en el medio y $34,2 \%$ en el bajo ${ }^{4}$. Estos resultados han sido comparados con los obtenidos por los hablantes de la norma culta (entrevistados hace ahora 30 años), que, en los casos seleccionados para el estudio de la atenuación, eran no solo personas con estudios universitarios, sino además profesionales de reconocido prestigio en la sociedad canaria (Samper Hernández, 2018); en ellos el porcentaje de recursos del dictum desciende al 20,3\%, lo que permite dibujar el perfil lineal representado en el Gráfico 4:

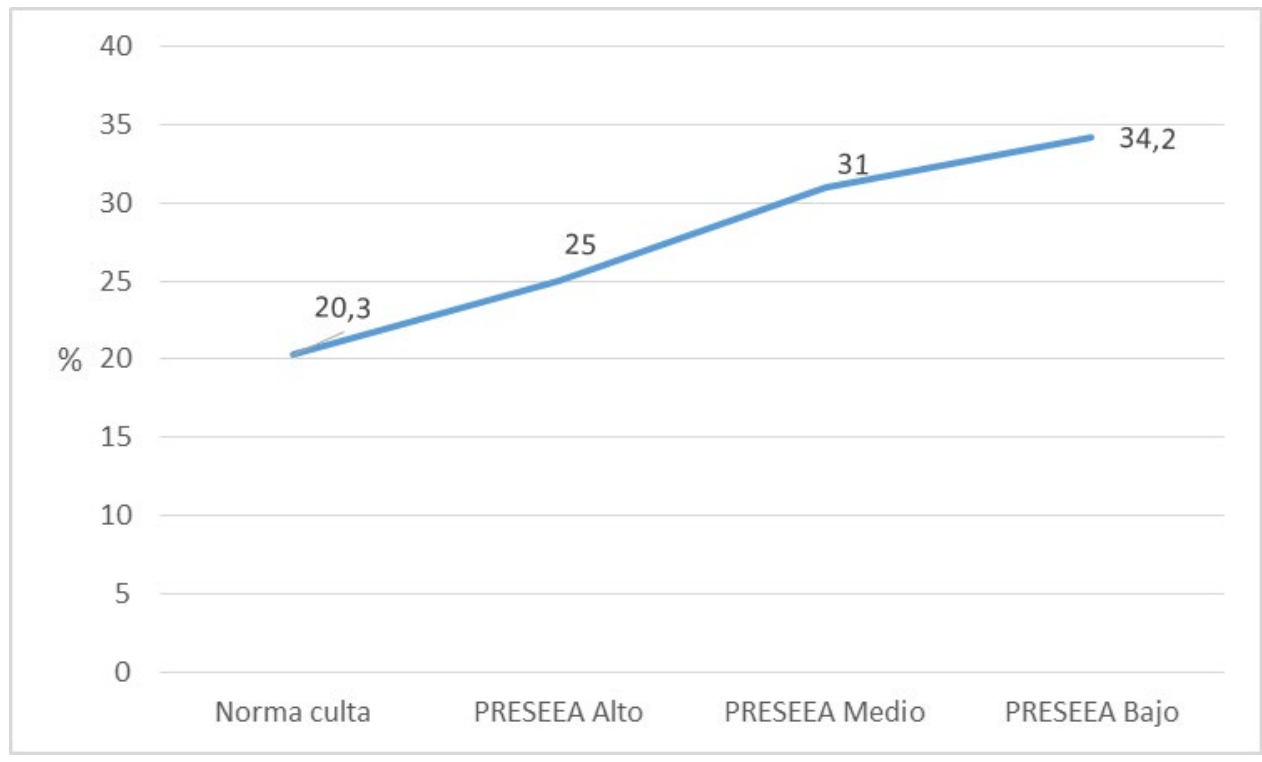

Gráfico 4. Recursos de atenuación del dictum según el nivel de instrucción. Datos de PRESEEA y Norma culta. 
La tendencia, pues, es clara: a medida que desciende el nivel de estudios, aumenta el empleo de los mecanismos de atenuación del dictum. Aunque la relación no es significativa desde el punto de vista estadístico (rho Spearman $=-0,016 ; \mathrm{p}>0,05$ ), se trata, sin duda, de un resultado relevante, que habrá de ser confirmado (o refutado) con una muestra más amplia.

\subsubsection{Edad}

Tabla 4. Recursos de atenuación empleados según la variable edad.

\begin{tabular}{|l|l|l|l|l|l|l|}
\hline \multirow{2}{*}{ Recursos } & \multicolumn{2}{|c|}{$\mathbf{1}$} & \multicolumn{2}{c|}{$\mathbf{2}$} & \multicolumn{2}{c|}{$\mathbf{3}$} \\
\cline { 2 - 8 } & $\mathbf{N}$ & $\mathbf{0}$ & $\mathbf{N}$ & $\mathbf{9}$ & $\mathbf{N}$ & \multicolumn{1}{|c|}{} \\
\hline Sufijos (diminutivos) & 28 & 7,8 & 26 & 7,7 & 11 & 5 \\
\hline Modificadores externos* & 62 & 17,3 & 51 & 15,1 & 21 & 9,6 \\
\hline Expresiones más suaves & 34 & 9,5 & 17 & 5 & 18 & 8,2 \\
\hline Extranjerismos & 0 & -- & 0 & -- & 0 & -- \\
\hline Tiempos verbales & 3 & 0,8 & 1 & 0,3 & 2 & 0,9 \\
\hline Aserciones como probabilidad/duda & 17 & 4,8 & 18 & 5,3 & 15 & 6,9 \\
\hline Fingimiento de ignorancia & 9 & 2,5 & 3 & 0,9 & 5 & 2,3 \\
\hline Acotación de la opinión & 17 & 4,8 & 17 & 5 & 14 & 6,4 \\
\hline Peticiones indirectas & 0 & -- & 0 & -- & 0 & -- \\
\hline Disculpas & 2 & 0,6 & 2 & 0,6 & 0 & -- \\
\hline Estructuras condicionales, concesivas o temporales & 3 & 0,8 & 10 & 3 & 6 & 2,7 \\
\hline Justificaciones o excusas & 33 & 9,2 & 30 & 8,9 & 29 & 13,2 \\
\hline Elipsis de la conclusión & 11 & 3,1 & 15 & 4,4 & 13 & 5,9 \\
\hline Impersonalizaciones & 24 & 6,7 & 25 & 7,4 & 12 & 5,5 \\
\hline Impersonalizaciones mediante el estilo directo* & 3 & 0,8 & 1 & 0,3 & 12 & 5,5 \\
\hline Reformulación & 24 & 6,7 & 30 & 8,9 & 9 & 4,1 \\
\hline Concesividad & 11 & 3,1 & 24 & 7,1 & 15 & 6,9 \\
\hline Partículas evidenciales* & 29 & 8,1 & 13 & 3,8 & 7 & 3,2 \\
\hline Elementos de control de contacto & 27 & 7,5 & 22 & 6,5 & 13 & 5,9 \\
\hline Formas de tratamiento y fórmulas apelativas* & 2 & 0,6 & 2 & 0,6 & 6 & 2,7 \\
\hline Elementos paralingüísticos, prosódicos y gestuales & 19 & 5,3 & 31 & 9,2 & 11 & 5 \\
\hline TOTALES & 358 & 100 & 338 & 100 & 219 & 100 \\
\hline
\end{tabular}

$*$ Resultados significativos $(\mathrm{p}<0,05)$

Existen, como vemos, distintas preferencias según las generaciones. El grupo de los mayores se distingue por ser el único que no presenta la modificación externa como recurso más frecuente $(9,6 \%$ frente al $17,3 \%$ y al 15,1\% de los jóvenes y adultos, respectivamente), un dato que resulta estadísticamente significativo $\left(\chi^{2}=12,323\right.$; $\mathrm{p}=0,002)$. En lugar de esta táctica, los hablantes de más edad recurren preferentemente a las justificaciones o excusas, que ocupan el tercer lugar en las listas de los dos grupos de contraste. Los sujetos jóvenes prefieren, tras la modificación externa, las expresiones más suaves, aspecto en el que se diferencian mucho de los hablantes adultos, que solo recurren a ellas en un $5 \%$ de las ocasiones en que atenúan. Estos últimos se decantan, en segundo lugar, por la recurrencia al paralenguaje $(9,2 \%)$, lo que los distingue de los jóvenes y de los mayores (ambos en torno a un 5\%).

Podemos afirmar, según estos datos, que existe una tendencia de los jóvenes a utilizar en mayor medida procedimientos para la atenuación del dictum, que suman el 
$34,6 \%$ del total de las tácticas registradas. Esto los diferencia de los adultos $(27,8 \%)$ y, más aún, de los mayores $(22,8 \%)$. Se constata, por tanto, una evolución ascendente, que resulta estadísticamente significativa (rho Spearman $=-0,176 ; \mathrm{p}<0,001$ ), del empleo de los recursos que afectan al contenido proposicional a medida que va descendiendo la edad de los sujetos, lo que permitiría plantear la hipótesis de que estos mecanismos serán cada vez más empleados, en sustitución tal vez de otros que afectan solo a la fuerza ilocutiva de los actos de habla. Esta hipótesis se vería reforzada, además, si consideramos los resultados obtenidos en el análisis de la atenuación de los informantes de la norma culta (con una atenuación general del dictum menor que la del grupo de más edad de PRESEEA), cuyas grabaciones fueron obtenidas alrededor del año 1990 y que, por lo tanto, permiten establecer comparaciones en tiempo real.

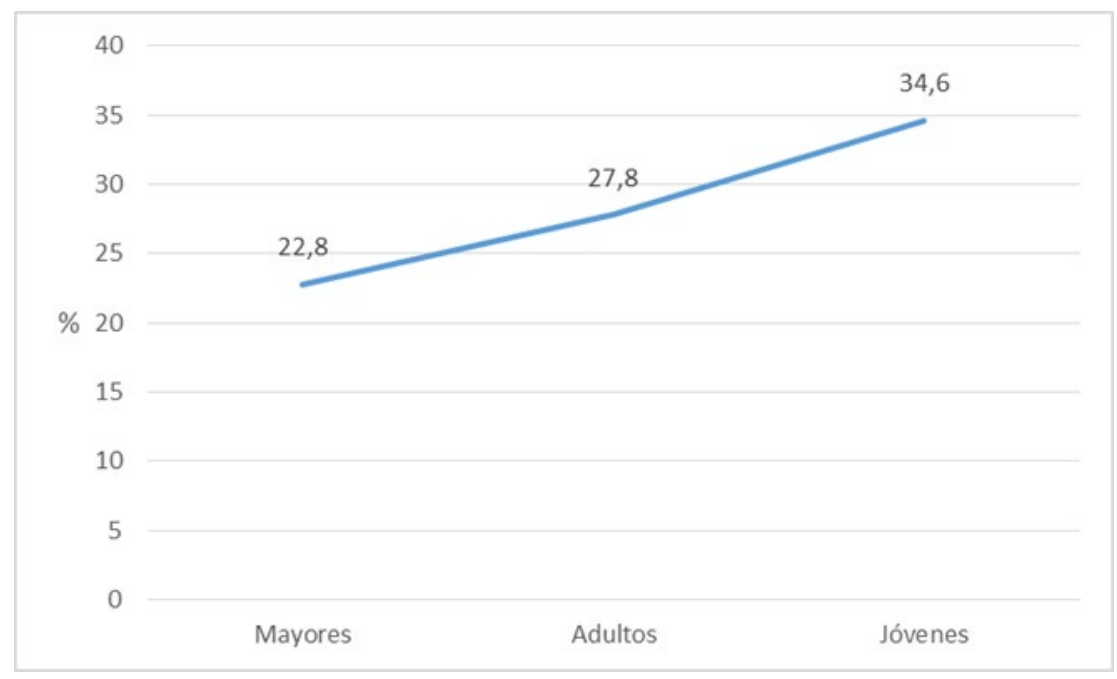

Gráfico 5. Recursos de atenuación del dictum según la variable generacional.

$\mathrm{Si}$ unimos los datos según el condicionante generacional con la tendencia que obteníamos en función del nivel de instrucción, se estaría dibujando el perfil de un cambio desde abajo, por el cual unos procedimientos que no son los preferidos por los niveles más altos del espectro se están extendiendo de forma inconsciente en toda la comunidad de habla palmense. El contar con materiales de la norma culta (grabados hace ahora 30 años) nos permite, además, confirmar este cambio con datos en tiempo real. 
Tabla 5. Recursos de atenuación empleados según la variable sexo.

\begin{tabular}{|l|l|l|l|l|}
\hline \multirow{2}{*}{ Recursos } & \multicolumn{3}{|l|}{ Hombres } & \multicolumn{3}{l|}{ Mujeres } \\
\cline { 2 - 6 } & $\mathrm{N}$ & $\%$ & $\mathrm{~N}$ & $\%$ \\
\hline Sufijos (diminutivos) & 25 & 5,5 & 40 & 8,6 \\
\hline Modificadores externos & 68 & 15 & 66 & 14,2 \\
\hline Expresiones más suaves & 36 & 8 & 33 & 7,1 \\
\hline Extranjerismos & 0 & -- & 0 & -- \\
\hline Tiempos verbales & 2 & 0,4 & 4 & 0,9 \\
\hline Aserciones como probabilidad o duda* & 34 & 7,5 & 16 & 3,5 \\
\hline Fingimiento de ignorancia & 12 & 2,7 & 5 & 1,1 \\
\hline Acotación de la opinión & 27 & 6 & 21 & 4,5 \\
\hline Peticiones indirectas & 0 & -- & 0 & -- \\
\hline Disculpas & 3 & 0,7 & 1 & 0,2 \\
\hline Estructuras condicionales, concesivas o temporales & 10 & 2,2 & 9 & 1,9 \\
\hline Justificaciones o excusas & 44 & 9,7 & 48 & 10,4 \\
\hline Elipsis de la conclusión & 20 & 4,4 & 19 & 4,1 \\
\hline Impersonalizaciones & 31 & 6,9 & 30 & 6,5 \\
\hline Impersonalizaciones mediante el estilo directo & 5 & 1,1 & 11 & 2,4 \\
\hline Reformulación & 34 & 7,5 & 29 & 6,3 \\
\hline Concesividad & 24 & 5,3 & 26 & 5,6 \\
\hline Partículas evidenciales & 23 & 5,1 & 26 & 5,6 \\
\hline Elementos de control del contacto & 33 & 7,3 & 29 & 6,3 \\
\hline Formas de tratamiento y fórmulas apelativas & 2 & 0,4 & 8 & 1,7 \\
\hline Elementos paralingüísticos, prosódicos y gestuales* & 19 & 4,2 & 42 & 9,1 \\
\hline Totales & 452 & 100 & 463 & 100 \\
\hline$*$ Resultados significativos (p < 0,05) & & & \\
\hline
\end{tabular}

En la Tabla 5 se observa que hombres y mujeres coinciden en gran medida en el empleo de los diferentes mecanismos atenuantes. En ambos casos, de acuerdo con los resultados generales, el recurso más utilizado es el de la modificación externa, seguido por la recurrencia a las justificaciones o excusas. Cabe destacar, sin embargo, tres diferencias fundamentales.

Por un lado, es notable la divergencia que existe entre los dos grupos con respecto al empleo de los elementos paralingüísticos (en nuestro caso, sobre todo las risas) como recursos atenuadores: en la lista femenina ocupan la tercera posición $(9,1 \%)$, mientras que en la masculina no aparecen hasta la duodécima (4,2\%). Se observa, pues, una mayor tendencia de las mujeres hacia el empleo de estos recursos paralingüísticos, una relación que resulta estadísticamente significativa $\left(\chi^{2}=8,483\right.$; $\mathrm{p}=0,004)$. Los datos de Las Palmas coinciden con los de Madrid y Santiago de Chile, si bien en estas dos comunidades no se dan diferencias abultadas, pero contrastan con los de Valencia, donde se observa un mayor uso de este mecanismo por parte de los hombres (Cestero et al., 2020).

Por otro lado, en la Tabla 5 llama la atención la frecuencia más elevada que alcanza la formulación de aseveraciones como probabilidad o duda en el caso de los hombres 
(con un total de 34 ocurrencias frente a los 16 de las entrevistadas, cifras que representan un $7,5 \%$ frente a un $3,5 \%$ en el conjunto de los recursos utilizados por cada grupo), un resultado estadísticamente significativo $\left(\chi^{2}=8,220 ; p=0,005\right)$ que contrasta con las tradicionales apreciaciones acerca de la mayor asertividad del habla masculina frente al habla femenina (por ejemplo, Lakoff, 1973; García Mouton, 1999). Se trata de un resultado que está en consonancia, sin embargo, con lo que sucede en la comunidad de habla madrileña (Cestero, 2017).

Por último, encontramos una diferencia que atañe a un mecanismo de atenuación del contenido proposicional: los diminutivos. Estos ocupan la cuarta posición en la nómina femenina $(8,6 \%)$ y no aparecen hasta la novena en la masculina $(5,5 \%)$. La diferencia en el empleo de la modificación interna va en consonancia con la idea de que la mujer utiliza con más frecuencia que el hombre los diminutivos, que constituirían un rasgo (proto)típicamente femenino. Ya en Samper Hernández (2019) apuntamos la frecuencia de uso muy superior de estas formas por parte de las encuestadas (que utilizan el 68,58\% de todos los registrados en el corpus), tanto si se empleaban con valor atenuador como si se utilizaban con otros valores como el afectivo, el peyorativo o el de disminución nocional. Era un resultado que coincidía, además, con el obtenido en otras comunidades que trabajan con los materiales de PRESEEA, como Madrid (Paredes, 2012) o Granada (Manjón-Cabeza, 2016). Ahora, atendiendo solo al valor atenuador, encontramos que en Las Palmas las entrevistadas registran el 61,5\% de los diminutivos, frente al 38,5\%, que son empleados por los hombres. Es decir, las diferencias, aunque ligeramente menores, se mantienen cuando se trata exclusivamente de los diminutivos con valor mitigador. El dato, aunque no resulta estadísticamente significativo $\left(\chi^{2}=3,101 ; \mathrm{p}=0,086\right)$, marca una tendencia que distingue a la comunidad de habla palmense de otras como la madrileña (Paredes, 2012), en la que los porcentajes de uso de hombres y mujeres son coincidentes cuando el diminutivo se emplea como mecanismo atenuante.

\section{CONCLUSIONES}

En este artículo se han presentado las funciones y los recursos de atenuación utilizados en 18 entrevistas del corpus PRESEEA-LP, así como las frecuencias absolutas y relativas que estos últimos alcanzan en relación con las variables sociales nivel de instrucción, edad y sexo. Creemos que se ofrece con ello una información que puede resultar de gran utilidad en la labor de comparación interdialectal que constituye uno de los objetivos principales del PRESEEA. El análisis de los datos se ha centrado en ciertos aspectos concretos que revelan determinadas tendencias propias del español de Las Palmas.

En lo que respecta a la función de la atenuación, se observa entre los hablantes palmenses un comportamiento general similar al que se encuentra en los corpus 
PRESEEA de Madrid-Vallecas, Santiago de Chile y Valencia, lo que estaría apuntando a una influencia determinante del tipo de género discursivo: las entrevistas semidirigidas, en las que predomina una finalidad transaccional (y no interpersonal), favorecen la aparición de la función autoprotectora de la imagen, que alcanza el 76\% en el corpus palmense. Del mismo modo, la función preventiva $(19,7 \%)$ busca sobre todo proteger la imagen de una tercera persona y no la del entrevistador.

Igualmente, en las cuatro modalidades contrastadas se da una atenuación mayoritaria de la fuerza ilocutiva del acto de habla de manera directa, aunque hay que señalar que en la comunidad canaria el uso de recursos mitigadores del contenido proposicional, aisladamente o en combinación con los que afectan al modus, se encuentra casi en el $40 \%$ de los actos de habla con atenuación.

A pesar de las semejanzas señaladas en las funciones y los tipos de atenuación, la comparación de las cuatro comunidades de habla evidencia también una mayor similitud de los resultados de Las Palmas con los de Santiago de Chile en ambos aspectos, lo que pone de manifiesto, una vez más, la cercanía entre el español de Canarias y el de América.

La revisión de los procedimientos de atenuación más utilizados en el corpus PRESEEA-LP revela la elevada frecuencia con que aparecen tres de los recursos mitigadores del dictum (modificadores externos, lítotes o eufemismos y modificadores internos), que suman casi el 30\% del total de los mecanismos empleados. Se trata de una característica diferencial con respecto a Madrid, Santiago de Chile y Valencia, que presentan porcentajes visiblemente más reducidos sobre todo en el uso de los diminutivos y de los mecanismos de selección léxica. Son resultados que revisten un gran interés por la información que pueden aportar acerca del diferente comportamiento dialectal en lo que respecta a la estrategia atenuadora (mitigación de la fuerza ilocutiva a través de la atenuación del propio contenido proposicional frente a atenuación directa de la fuerza ilocutiva), y por ello se han convertido en el foco principal de este trabajo.

El análisis de los recursos de atenuación a la luz de las variables nivel de instrucción y edad dibuja el perfil de un cambio desde abajo, por el cual los citados procedimientos de atenuación del dictum, que no son los preferidos por los niveles más altos del espectro social insular, se están extendiendo de forma inconsciente en la comunidad de habla palmense. Entre los hablantes jóvenes la suma de estos mecanismos supone el 34,6\% de todos los encontrados; podemos pensar, por tanto, que el incremento total de la actividad atenuadora que había observado Samper Hernández $(2017,2018)$ entre los sujetos de la primera generación se debe fundamentalmente al mayor uso de los recursos de atenuación del dictum. Este cambio vendría impulsado sobre todo por los hablantes jóvenes de los niveles de instrucción bajo y, en menor medida, medio. Se trata, sin duda, de un aspecto interesante y 
posiblemente diferencial del español isleño, en el cual habrá que seguir profundizando en futuros trabajos.

En lo que respecta al factor sexo, pueden señalarse tres aspectos diferenciales entre la actividad atenuadora de hombres y mujeres: en primer lugar, la mayor tendencia de las encuestadas al empleo de las risas y la entonación, que coincide con lo que se observa otras comunidades de habla como Madrid o Santiago de Chile; en segundo lugar, la preferencia de los hombres por las aserciones en forma de duda, lo que iría en contra de la concepción tradicional sobre el carácter más asertivo del habla masculina; por último, el mayor uso del diminutivo con valor atenuador por parte de las entrevistadas, lo que vendría a corroborar el carácter prototípicamente femenino de este sufijo también cuando se usa con valor atenuador en Las Palmas de Gran Canaria, algo que distinguiría esta comunidad de habla de otras como la madrileña y que podría estar apuntando a una actitud negativa de los hombres palmenses hacia esta sufijación, independientemente de su valor en el discurso.

\section{REFERENCIAS BIBLIOGRÁFICAS}

Acín, E. (2017). Atenuación y discurso académico: Las Conclusiones de la tesis de doctorado. LEA: Lingüistica Española Actual, 39(2), 153-175.

Albelda, M. (2011). Variación sociolingüística en las estrategias de atenuación del corpus PRESEEA-Valencia del sociolecto alto. En A. M. Cestero, I. Molina \& F. Paredes (Eds.), La lengua, lugar de encuentro. Actas del XVI Congreso Internacional de la Alfal (pp. 1857-1866). Alcalá de Henares: Universidad de Alcalá.

Albelda, M. (2012). Estudio sociolingüístico (piloto) de las estrategias de atenuación en el corpus PRESEEA de Valencia. En E. Ridruejo (Coord.), Tradición y progreso en la lingüistica general (pp. 9-28). Valladolid: Universidad de Valladolid.

Albelda, M. (2013). La atenuación: Tipos y estrategias. En J. R. Gómez Molina (Ed.), El español de Valencia. Estudio sociolingüistico (pp. 315-343). Frankfurt: Peter Lang.

Albelda, M. (2016). Sobre la incidencia de la imagen en la atenuación pragmática. Revista Internacional de Lingüistica Iberoamericana, 27(1), 19-32.

Albelda, M. (2018). Variación sociolingüística de los mecanismos mitigadores: Diferencias de uso en edad y sexo. Cultura, Lenguaje y Representación, 19, 7-29.

Albelda, M. \& Briz, A. (2010). Aspectos pragmáticos. Cortesía y atenuantes verbales en las dos orillas a través de muestras orales. En M. Aleza \& J. M. Enguita (Coords.), La lengua española en América: Normas y usos actuales (pp. 237-260). Valencia: Universitat de València. 
Albelda, M., Briz, A., Cestero, A. M., Kotwica, D. \& Villalba, C. (2014). Ficha metodológica para el análisis pragmático de la atenuación en corpus discursivos del español (ES.POR.ATENUACIÓN). Oralia, 17, 7-62.

Albelda, M. \& Mihatsch, W. (Eds.) (2017). Atenuación e intensificación en géneros discursivos. Madrid/Frankfurt: Iberoamericana/Vervuert.

Barros, M. J. (2017). Funciones de la atenuación en la conversación coloquial entre jóvenes del Sur de España. Lingüistica Española Actual, 39(2), 199-216.

Béjar, R. (2015). Estudio del fenómeno de la atenuación en la ciudad de Granada (nivel sociocultural alto), Revista Electrónica del Lenguaje, 1.

Briz, A. (2007). Para un análisis semántico, pragmático y sociopragmático de la cortesía atenuadora en España y América. Lingüistica Española Actual, 29(1), 540 .

Briz, A. \& Albelda, M. (2013). Una propuesta teórica y metodológica para el análisis de la atenuación lingüística en español y portugués. La base de un proyecto en común (ES.POR.ATENUACIÓN). Onomázein, 28, 288-319.

Cestero, A. M. (2011). Las estrategias de atenuación: Estudio sociolingüístico. En E. Ridruejo, N. Mendizábal, C. Vela et al. (Eds.), Actas del IX Congreso Internacional de Lingüistica General (pp. 525-542).Valladolid: Universidad de Valladolid.

Cestero, A. M. (2012). Recursos lingüísticos de atenuación en el habla de Madrid. Estudio sociopragmático. En T. Jiménez Juliá, B. López Meirama, V. Vázquez \& A. Veiga (Eds.), Cum corde et in nova grammatica. Estudios ofrecidos a Guillermo Rojo (pp. 233-246). Santiago de Compostela: Universidad de Santiago de Compostela.

Cestero, A. M. (2015). La atenuación lingüística en el habla de Madrid: Un fenómeno sociopragmático variable. En A. M. Cestero, I. Molina \& F. Paredes (Eds.), Patrones sociolingüisticos de Madrid (pp. 365-412). Berna: Peter Lang.

Cestero, A. M. (2017). La atenuación en el habla de Madrid: Patrones sociopragmáticos. RILCE: Revista de Filología Hispánica, 33(1), 57-86.

Cestero, A. M. \& Albelda, M. (2012). La atenuación lingüística como fenómeno variable. Oralia, 15, 77-124.

Cestero, A. M. \& Rodríguez Alfano, L. (2014). Guía de estudio de la atenuación en los corpus PRESEEA [en línea]. Disponible en: http://preseea.linguas.net/LinkClick.aspx?fileticket=rkX99uxr6pI\%3d\&tabid $\equiv 92 \& \mathrm{mid}=497$ 
Cestero, A. M., Albelda, M., Guerrero, S. \& Samper Hernández, M. (2020). Variación sociopragmática y geolectal en el uso de atenuación. Lengua y Habla, 24, 1-53.

Chao, M. (2018). Procedimientos de atenuación en los artículos de investigación científica: Las directrices cognitivas en español. En A. García Ramón \& M. A. Soler (Eds.), ELUA: Estudios de Lingüistica. Universidad de Alicante. $N^{0}$ Extra 4: Estudios de atenuación en el discurso, 139-156.

Contreras, J. (2018). La impersonalidad como estrategia de atenuación en la novela 'El lector de Julio Verne’ de Almudena Grandes: un análisis contrastivo alemán / español. RILCE: Revista de Filología Hispánica, 34(3), 1243-1258.

Douglas, S., Soler, M. A. \& Vuoto, J. (2018). La atenuación en conversaciones coloquiales argentinas y españolas: Un estudio contrastivo. RILCE: Revista de Filología Hispánica, 34(3), 1280-1312.

Estellés, M. (2013). La expresión fónica de la cortesía en tribunales académicos. Las intervenciones en tribunales de tesis y trabajos de investigación. Oralia, 16, 81110.

Estellés, M. \& Cabedo, A. (2017-2018). La atenuación fónica en entrevistas (proyecto PRESEEA) y en conversaciones (corpus Val.Es.Co): Un estudio de campo. Linred, 15.

Flores, M. E. (2016). Atenuación e intensificación en el macro acto de ironizar en entrevistas orales. Letrónica, 9(1), 37-48.

Flores, M. E. (2017). La atenuación y los marcadores de foco de exclusión en los corpus: PRESEEA-Mty / AMERESCO-Méx.Mty. Normas, 7, 19-32.

Flores, M. E. (2019). Variación regional en el empleo del marcador de foco de exclusión nomás en el español hablado de México. En M. E. Placencia \& C. Fuentes (Coords.), Sección "Variación regional en el uso de marcadores del discurso en español”, Revista Internacional de Lingüistica Iberoamericana, (XVII), 1 (33), 37-56.

García Mouton, P. (1999). Cómo hablan las mujeres. Madrid: Arco Libros.

Grupo Val.Es.Co. (2014). Las unidades del discurso oral. La propuesta Val.Es.Co. de segmentación de la conversación (coloquial). Estudios de Lingüistica del Español, 35, 13-73.

Guerrero, S. (En prensa). La atenuación lingüística en el corpus PRESEEA de Santiago de Chile. Alpha, 51(1).

Haverkate, H. (1994). La cortesía verbal: Estudio pragmalingüistico. Madrid: Gredos. 
Kern, B. \& Villalba, C. (2017). Apelación y atenuación: Comparación intergenética entre juicios orales y debates parlamentarios españoles. Revista de Lingüística Teórica y Aplicada, 55(2), 169-195.

Lakoff, R. (1973). Language and woman's place. Language in society, 2(1), 45-80.

Manjón-Cabeza, A. (2016). Los sufijos -ico, -ito, -illo en el español de Granada, España. Spanish in Context, 13(3), 420-435.

Mihatsch, W. \& Albelda, M. (Coords) (2016). La atenuación y la intensificación desde una perspectiva semántico-pragmática. Revista Internacional de Lingüistica Iberoamericana, 14(27), 7-153 sección temática.

Molina, I. (2005). Estrategias de atenuación en el habla de las mujeres madrileñas (barrio de Salamanca de Madrid). Documentos de Español Actual, 6-7, 89-100.

Molina, I. (2015). Estrategias de atenuación en el Barrio de Salamanca de Madrid. En A. M. Cestero, I. Molina \& F. Paredes (Eds.), Patrones sociolingüísticos de Madrid (pp. 349-364). Berna: Peter Lang.

Montoro, E. (2014). Recursos de atenuación en el corpus PRESEEA-Granada: Estudio del sociolecto alto. Comunicación presentada en el XVII Congreso Internacional de la ALFAL, Universidad de Joao Pessoa, Paraíba, Brasil.

Moreno, F. (2009). La lengua española en su geografía. Madrid: Arco Libros.

Palacios, N. (2017). Estrategias y mecanismos de atenuación en PRESEEA-Puebla: Instrucción educativa baja. Anuario de Letras: Lingüistica y Filología, 5(2), 149182.

Paredes, F. (2012). Variación en el uso del diminutivo en el habla de Madrid: Avance de un estudio sociolingüístico. En A. M. Cestero, I. Molina \& F. Paredes (Eds.), La lengua, lugar de encuentro. Actas del XVT Congreso Internacional de la ALFAL, edición en CD (pp. 3709-30719). Alcalá de Henares: Servicio de Publicaciones de la Universidad de Alcalá.

Repede, D. \& León-Castro, M. (2019). Procedimientos de impersonalización en la ciudad de Sevilla: El caso del pronombre uno. Cuadernos de Investigación Filológica, 46, 81-106.

Samper Hernández, M. (2013). La atenuación lingüística en el español de Las Palmas de Gran Canaria. Lingüistica Española Actual, 35(2), 325-348. 
Samper Hernández, M. (2017). Análisis sociolingüístico de la atenuación en el español de Las Palmas de Gran Canaria. En M. Albelda \& W. Mihatsch (Eds.), Atenuación e intensificación en géneros discursivos (pp. 153-168). Madrid-Frankfurt: Iberoamericana-Vervuert.

Samper Hernández, M. (2018). Un cambio en tiempo real: La atenuación entre hablantes universitarios de Las Palmas de Gran Canaria. RILCE: Revista de Filología Hispánica, 34(3), 1259-1279.

Samper Hernández, M. (2019). Aproximación al uso del diminutivo en Las Palmas de Gran Canaria: Datos sociolingüísticos a partir de muestras orales de actuación. En A. Cabedo \& A. Hidalgo (Eds.), Pragmática del español hablado. Hacia nuevos horizontes (pp. 433-445). Valencia: Universitat de Valéncia.

Samper Padilla, J. A. \& Samper Hernández, M. (2020). The weakening of intervocalic /d/ in the Spanish of Las Palmas de Gran Canaria: Comparison with other Speech Communities, Spanish in Context, 17(2), 221-246.

Silva-Corvalán, C. \& Enrique-Arias, A. (2017). Sociolingüistica y pragmática del español. Washington DC: Georgetown University Press.

Soler, M. A. (2018). Fingimientos y atenuación en el uso de 'creo'. RILCE: Revista de Filología Hispánica, 34(3), 1104-1128.

Torres, Y. \& Rodríguez, Y. (2017). La atenuación en Barranquilla: Estudio sociopragmático. Cuadernos de Lingüistica Hispánica, 30, 55-79.

Villalba, C. (2016). Actividades de imagen, atenuación e impersonalidad en los juicios orales. Tesis doctoral, Universitat de València, València, España.

Villalba, C. (2018). El infinitivo enunciativo como estrategia atenuante. Un estudio a partir de juicios orales. Spanish in Context, 15(2), 237-257.

\section{NOTAS}

${ }^{1}$ Esta publicación forma parte de los proyectos de investigación Estudio complementario de los patrones sociolingüísticos del español de Las Palmas de Gran Canaria (ECOPASOS-LPGC) (Referencia FFI2015-68171-C5-5-p), financiado por el Ministerio de Economía y Competitividad y subvencionado con fondos FEDER, y Agenda 2050. El español de Sevilla y Las Palmas de Gran Canaria: procesos de variación y cambio espaciales y sociales (Referencia PID2019-104982GB-C54), financiado por el Ministerio de Ciencia e Innovación.

2 Para una mayor información, véase Cestero y Rodríguez Alfano (2014). 
3 Tras cada ejemplo se registra el código de la ciudad (LP), el número de la entrevista, el sexo del informante (H o M), su edad (generaciones 1, 20-34 años; 2, 35-54 años; y 3, 55 años o más) y su nivel educativo (1, primario; 2 , secundario; y 3 , universitario).

${ }^{4}$ De hecho, los hablantes de instrucción primaria son los que presentan una mayor proporción de atenuación exclusiva del dictum (25,5\%, frente al 21,7\% y al escaso $14,6 \%$ de los hablantes del nivel medio y del universitario, respectivamente). 